Voix et Images

voixetimages

\title{
Écrire-traduire entre les langues
}

Les effets de traduction et de bilinguisme dans les romans de

Gail Scott

Writing/Translating Between Languages

Effects of Translation and Bilingualism in the Novels of Gail

Scott

\section{Gillan Lane-Mercier}

Volume 30, numéro 3 (90), printemps 2005

La littérature anglo-québécoise

URI : https://id.erudit.org/iderudit/011859ar

DOI : https://doi.org/10.7202/011859ar

Aller au sommaire du numéro

Éditeur(s)

Université du Québec à Montréal

ISSN

0318-9201 (imprimé)

1705-933X (numérique)

Découvrir la revue

Citer cet article

Lane-Mercier, G. (2005). Écrire-traduire entre les langues : les effets de traduction et de bilinguisme dans les romans de Gail Scott. Voix et Images, 30(3), 97-112. https://doi.org/10.7202/011859ar
Résumé de l'article

Cet article propose, dans une perspective traductologique, une réflexion sur la traduction et le bilinguisme comme effets d'écriture dans les romans de Gail Scott. Définis comme expérience quotidienne, comme pratique auto-réflexive et comme trope, les effets de traduction et de bilinguisme sont tributaires à la fois de l'anglais " minorisé » employé par Scott et de la présence de fragments - voire de passages entiers - rédigés en français. Il s'agit d'examiner les modes d'inscription de ces effets, les images/valeurs qu'ils véhiculent, ainsi que les postures esthétiques, idéologiques et éthiques qu'ils présupposent face à l’altérité linguistique et culturelle. 
ÉCRIRE-TRADUIRE ENTRE LES LANGUES.

Les effets de traduction et de bilinguisme dans les romans de Gail Scott

$$
++
$$

\section{GILLIAN LANE-MERCIER}

Université McGill

\section{RÉSUMÉ}

Cet article propose, dans une perspective traductologique, une réflexion sur la traduction et le bilinguisme comme effets d'écriture dans les romans de Gail Scott. Définis comme expérience quotidienne, comme pratique auto-réflexive et comme trope, les effets de traduction et de bilinguisme sont tributaires à la fois de l'anglais "minorisé » employé par Scott et de la présence de fragments - voire de passages entiers - rédigés en français. Il s'agit d'examiner les modes d'inscription de ces effets, les images/valeurs qu'ils véhiculent, ainsi que les postures esthétiques, idéologiques et éthiques qu'ils présupposent face à l'altérité linguistique et culturelle. 
La traduction joue un rôle primordial dans les essais et romans de l'écrivaine anglomontréalaise Gail Scott qui inaugure, avec la publication de Heroine en 1987, une nouvelle tendance au sein des lettres canadiennes: le roman bilingue anglaisfrançais. À la fois une expérience quotidienne, une pratique autoréflexive, une stratégie d'écriture et un trope, la traduction creuse des espaces textuels poreux, mouvants, ambigus, où les identités culturelles fléchissent et les différences linguistiques s'éprouvent selon une dynamique du risque, de la mise à l'épreuve, de la souffrance, de la (re)connaissance et du partage. Les romans de Scott s'inscrivent dans un projet esthétique et politique de "minorisation ${ }^{1}$ » de l'anglais visant, d'une part, à capter, dans la texture même de l'écriture, leur ancrage dans des lieux et une époque où «les identités "locales" sont à tout moment sollicitées et pénétrées par diverses formes d'altérité culturelle ${ }^{2}$ ", et, d'autre part, à interroger les multiples postures esthétiques, idéologiques et éthiques qu'il est possible d'adopter face à cette altérité, ainsi que les modalités de négociation et d'accommodement que ces postures impliquent. Ce faisant, ils dénoncent, par le biais d'effets de traduction et de bilinguisme soigneusement aménagés, tant les inégalités inhérentes au pluralisme libéral et au bilinguisme officiel qui en découle, que les nivellements identitaires opérés au nom de l'égalité par l'idéologie républicaine. Dans une perspective traductologique qui privilégie leur double statut sémiotique et éthique, une réflexion sur les effets de traduction et de bilinguisme dans les trois romans de Scott s'impose; elle sera suivie d'une analyse plus détaillée de son dernier roman, $M y$ Paris. A Novel, publié en 1999.

\section{QUELQUES PROPOSITIONS D'ORDRE THÉORIQUE}

Que ces effets de traduction et de bilinguisme soient indissociables d'une autoréflexivité implicite ou explicite ne fait aucun doute; dès 1986, Scott note dans un essai intitulé «A Visit to Canada» que la duplicité culturelle, c'est-à-dire «la traduction constante des idées du français à l'anglais dans mon écriture, de l'anglais au français dans mes paroles» rend «nécessaire l'auto-réflexivité pour que je ne me noie pas dans la confusion ${ }^{3}$ » créée par le choc des différences. Synonyme de démission, d'assimilation et, ultimement, de disparition, ce danger de noyade l'amène à refuser

$$
++
$$

1 Les termes de «minorisation» et, plus loin, de «minorisé» sont calqués sur celui de «minoritizing" proposé par Lawrence Venuti, «Translation, Heterogeneity, Linguistics», TTR, vol. IX, nº 1, 1996, p. 91-115. 2 Sherry Simon, Le trafic des langues: traduction et culture dans la littérature québécoise, Montréal, Boréal, 1994, p. 12. 3 Traduction de: «the endless translation of ideas in my writing from French to English, in my speech from English to French», rend "self-reflexiveness necessary in order not to drown in [the] confusion» (Gail Scott, "A Visit to Canada", Spaces Like Stairs: Essays, Toronto, The Women's Press, 1989, p. 47). Toutes les traductions de Scott sont de moi. Désormais, les références à cet ouvrage seront indiquées par le sigle SS, suivi du folio, et placées entre parenthèses dans le texte. 
la conception, alors dominante au Canada anglais, de la traduction comme pont entre les deux langues-cultures fondatrices et du bilinguisme garant d'unité nationale:

In the function of bridge or go-between, there is no equitable give-and-take. [...] the messenger [is] forgotten in the process. [...] people in English Canada often thought of me as having precisely the same culture as themselves. Their not sensing my difference [...] reflects the arrogant assumptions of any dominant culture: i.e., that a minority culture (Québec) does not have the vitality to operate as a sphere of influence on those "minorities" (anglophones) that live within its parameters. [...] to suppress the awareness of [...] difference, if one speaks from a minority position, is to risk the drowning of that difference in the expectations of the dominant other ${ }^{4}$. (SS, 48-49)

Ce refus des tropes de la traduction comme pont et du traducteur comme passeur désincarné - sans corps, ni voix, ni identité - de contenus culturels accessibles et assimilables coïncide avec le projet d'écriture de Scott, qu'elle définit comme «un désir de déplacer le discours, de sortir des cadres, plutôt que de parler depuis leur centre ${ }^{5}$ » et un moyen de déconstruire les images prescrites, stéréotypées, de soi et de l'autre en y pratiquant des «trous» dans lesquels le sujet traduisantécrivant fait résonner sa voix (sa différence) et où le sujet lisant-traduisant est appelé à reconnaître sa propre altérité et à participer à la production de nouvelles images. Plutôt que de chercher, selon une logique reterritorialisante fondée sur l'analogie, la clôture et la perte, à réduire les écarts culturels afin d'assurer le maximum de compréhension chez l'autre, il s'agit d'investir, "sans jamais prétendre le fermer ${ }^{6}$ » (SS, 47), cet espace mitoyen et mouvant entre deux manières de penser, en vue de constituer "une suite d'égalités qui transforme la perte [...] (il y a toujours, inévitablement, des pertes) en richesse ${ }^{7}$ » $(S S, 54)$ par le truchement $\mathrm{d}^{\prime}$ «un sujet du roman qui tente de parler de deux pôles opposés simultanément ${ }^{8} 》(S S, 87)$.

Car vivre-traduire-écrire entre l'anglais et le français au Québec, c'est «voir à double sens ${ }^{9}$ » et assister au brouillage des repères identitaires, langagiers et

$$
+++
$$

4 «La fonction de pont ou d'intermédiaire exclut tout échange équitable. [...] on oublie le messager [...] au cours du processus. [...] au Canada anglais, les gens estimaient souvent que je possédais exactement la même culture qu'eux. Le fait qu'ils n'aient pas senti ma différence [...] reflète l'arrogance des idées reçues de toute culture dominante, à savoir qu'une culture minoritaire (le Québec) n'a pas la vitalité nécessaire pour fonctionner comme sphère d'influence sur les "minorités" (anglophones) qui habitent à l'intérieur de ses frontières. [...] supprimer toute conscience de la différence [...], lorsqu'on parle d'une position minoritaire, c'est courir le risque de noyer cette différence dans les attentes de l'autre hégémonique.» $\mathbf{5}$ Traduction de: "a desire to displace discourse, step out of frames, rather than speak within them» (Gail Scott, «"a very rhythmic and almost conversational style"», Janice Williamson (dir.), Sounding Differences. Conversations with Seventeen Canadian Women, Toronto, University of Toronto Press, 1993, p. 255). 6 Traduction de: «never pretending to close it». 7 Traduction de: «a string of equalities that transforms [...] loss (there is always inevitably loss) into wealth». 8 En français dans le texte. C'est Scott qui souligne. 9 Gail Scott, «Mon Montréal: notes d'un écrivain anglophone», Marie-Andrée Beaudet (dir.), Échanges culturels entre les «Deux solitudes», Sainte-Foy, Presses de l'Université Laval, 1999, p. 95. Désormais, les références à cet article seront indiquées par le sigle $M O N$, suivi du folio, et placées entre parenthèses dans le texte. 
culturels en adoptant une posture de doute face à l'écriture qui récuse les certitudes, ébranle les rapports de domination et rend caduque l'opposition entre soi et l'autre. Désormais érigée en trope privilégié de l'intermédialité - voire de la modernité tout court ${ }^{10}-$, la traduction s'avère la voie d'accès à l'invention d'une manière d'être et d'un style d'écriture inédits, minorisés, bilingues, aptes à dénoter ce lieu où langue maternelle et langue du texte, «trouée[s] par la présence de l'autre» $(M O N, 100)$, dérapent face à la langue française, où sujet parlant, sujet écrivant et sujet lisant deviennent poreux et où, du coup, les différences culturelles se négocient à partir de bases nouvelles: «Là où s'ouvre un vide se présente une occasion d'explorer» (MON, 100), donc de (se) (re)créer.

L'écriture romanesque de Scott est une recherche formelle dont les soubassements théoriques se manifestent en grande partie par des effets de traduction et de bilinguisme à forte charge autoréflexive. Tantôt le français s'insinue, selon un processus traductif implicite opéré au préalable par le sujet traduisant-écrivant, dans les rythmes, tonalités et constructions de sa prose en y agençant des aires de contact interlinguistique où prime, au-delà de la perméabilité et de l'interpénétration, un usage proprement intensif (mineur, déterritorialisé) de l'anglais; tantôt il fait brusquement irruption entre deux mots, phrases ou paragraphes en y introduisant des espaces d'altérité «brute» à dominante performative, destinés à engager directement le lecteur anglophone dans un processus traductif plus ou moins hasardeux; tantôt, enfin, il s'accompagne de son "équivalent» anglais au moyen d'un processus traductif "simultané» qui ouvre un lieu de cohabitation linguistique et culturelle moins aliénant et, par là, potentiellement plus égalitaire.

Mais c'est moins la nature des modalités d'inscription qui importe que les valeurs esthétiques, idéologiques et éthiques qu'elles véhiculent. Non seulement Scott «cherche à travailler avec le français à l'oreille [... pour] échapper à certains tics de sa propre culture» (MON, 94), et ainsi "maintenir une certaine distance critique par rapport à la représentation de sa [...] langue/culture ${ }^{11}{ }^{\prime}$, mais elle se sert du français pour procéder à une critique virulente du postulat d'analogie inhérent aux discours culturels hégémoniques, notamment ceux préconisant la construction de ponts: «A text [...] carrying an unacknowledged cultural difference in the country called Canada can't help but implicitly challenge certain assumptions about language (and about Canadian history) held by most anglophones, whose relationship to language is not, historically, one of oppression ${ }^{12}$.» (SS, 53) D'un côté, la présence du français dans ses romans permet d'exposer le caractère idéaliste et

$+++$

10 Voir Linda Leith, «Face to Face. An Interview With Gail Scott», Matrix, n 28, 1989, p. 24. «I think this influence of one language on another is perfectly in keeping with the idea of modernité.» ("Je pense que cette influence d'une langue sur une autre est parfaitement en accord avec l'idée de la modernité.») 11 Traduction de: «narratively frame her own language/culture from a certain critical distance» (Gail Scott, «My Montréal. Notes of an Anglo-Québécois Writer», Brick, n 59, 1998, p. 5). Désormais, les références à cet article seront indiquées par le sigle $M Y$, suivi du folio, et placées entre parenthèses dans le texte. $\mathbf{1 2}$ «Un texte [...] qui véhicule une différence culturelle non reconnue au pays qu'on appelle le Canada ne peut que contester implicitement certaines idées reçues concernant la langue (et l'histoire du Canada) partagées par la plupart des anglophones, dont le rapport à la langue ne relève pas, historiquement, de l'oppression.» 
élitiste du bilinguisme officiel qui continue à servir les institutions politiques et culturelles canadiennes en reconduisant, grâce à la traduction conçue comme pont, le monolinguisme et le monoculturalisme qu'il est censé combattre. De l'autre côté, son anglais minorisé, "fortement imprégné de la culture et de la langue majoritaire franco-québécoise ${ }^{13}$ ", fait ressortir à l'intention de ses lecteurs anglophones le caractère hétéroglossique, pluriel, instable de leur langue qui, au Québec, a perdu son statut de langue majeure, et la spécificité tonale de l'anglo-québécois. L'illusion monolithique sous-jacente aux langues-cultures dominantes, de même qu'à un certain type de pratique traductionnelle axée sur «la transmission forcée des cultures, langues ${ }^{14}{ }^{1}$, est à tour de rôle interrogée, problématisée, tournée en dérision et dépassée pour que se fasse entendre un sujet traduisant-écrivant angloquébécois qui, ironiquement, écrit et n'écrit pas en anglais ${ }^{15}$ :

This posture of acknowledging difference at the point where difference meets is, increasingly, for me, the posture of the juggler or clown. At first bowing this way and that. But little by little, refining, [...] until, with a minimum of words or gestures, she speaks to the maximum of people. It is a game [...] of eternal distance and bemusement ${ }^{16}$. $(M Y, 7)$

Tributaires d'une volonté autoréflexive de dénoter l'intermédialité, les effets de traduction et de bilinguisme dans les romans de Scott sont également redevables d'un processus d'écoute de la ville qu'elle habite et des langues qui s'y déploient, avec leurs sonorités, rythmes, densités, inflexions, tensions. Véritable cacophonie de fond qu'il s'agit de capter dans sa matérialité, ces tonalités porteuses d'altérité incitent Scott à établir un lien direct entre sujet traduisant-écrivant, texte et contexte:

It's very possible the sound-effects that trouble my narrative and even my syntax [...] are, among other things, a formal response to the question of how to best represent my city. Its pulses, its tensions. Its ceaseless plethora of strong minority voices $[\ldots]$ constantly challenging any notion of authority ${ }^{17}$. $(M Y, 5)$

$$
+++
$$

13 Gail Scott, «Miroirs inconstants», Quebec Studies, n 26, 1998-1999, p. 23.14 Traduction de: «the forced transiting of cultures, tongues» (Louise Cotnoir, Barbara Godard, Susan Knutson, Daphne Marlatt, Kathy Mezei et Gail Scott, «Introduction: Women of Letters», Barbara Godard (dir.), Collaboration in the Feminine. Writings on Women and Culture from Tessera, Toronto, Second Story Press, 1994, p. 18). 15 Voir Linda Leith, op. cit., p. 24. «I do and I don't write in English.» 16 «Pour moi, cette posture qui consiste à reconnaître la différence au point où les différences se rencontrent devient de plus en plus celle du jongleur ou de la clown. D'abord, elle fait des révérences à gauche, à droite. Mais peu à peu, grâce à des raffinements, [...] elle parvient à s'adresser à un maximum de personnes avec un minimum de mots ou de gestes. C'est un jeu [...] de distanciation et de stupéfaction sans fin.» 17 «Il est fort possible que les effets sonores qui perturbent mes récits et même ma syntaxe [...] soient, entre autres, une réponse formelle à la question de savoir comment représenter le mieux ma ville. Ses pouls, ses tensions. Sa pléthore incessante de voix minoritaires fortes [...], sans cesse contestant toute notion d'autorité.» 
D’où un déplacement intéressant de l'enjeu initial. Si, d'un point de vue idéologique et éthique, les effets de traduction minent les ponts, évacuent le principe d'analogie et font du texte un lieu mitoyen où les différences se risquent et s'assument, d'un point de vue esthétique (formel), ils réactivent le principe d'analogie en faisant du texte un lieu poreux où les différences linguistiques et culturelles s'organisent en réseaux de ressemblances: "Listening ultimately permits a letting go of the strict boundaries of identity; self can drift into context ${ }^{18} . »(M Y, 7)$

Les intrusions indirectes et directes du français dans les romans de Scott possèdent donc, outre leur dimension autoréflexive, une importante visée référentielle ou d'authentification ${ }^{19}$. Si Scott recourt au français, $c^{\prime}$ est tout simplement parce que la plupart de ses personnages parlent français et que ses narratrices anglophones "découvrent qu'il y a des choses pour lesquelles elles ne trouvent pas d'équivalent en anglais ${ }^{20}$ ». Si elle utilise un anglais minorisé, c'est pour simultanément mieux exprimer le Montréal contemporain et forger «une voix qui soit aussi proche que possible de ma propre expérience, une voix qui reflète, dans la texture même du texte, dans la sonorité des phrases et de la langue, le lieu où les cultures se croisent $^{21}$.» Cela lui permet aussi de mieux construire ses narratrices, surtout celle de Heroine, soucieuse «de soulever la question de savoir comment l'héroïne anglophone (d'un roman) serait perçue dans le contexte [de la société] québécois[e] contemporain[e] ${ }^{22}$ », à laquelle elle appartient et n'appartient pas ${ }^{23}$. Ces clivages linguistiques et culturels, tant internes - car constitutifs du sujet traduisantécrivant bilingue - que contextuels, requièrent un style "glissant", à la fois autoréflexif pour éviter la confusion du choc des différences et référentiel pour être en mesure de l'exprimer: "I am beginning to see, note Scott à propos de la même narratrice, what her internal grammar does to the sentence, the paragraph, how the paragraphs "slip", and the sentences, to reflect this turbulent inner resonance ${ }^{24}$.» (SS, 89) Scott précise ailleurs: "One just has to get over that fear of sounding... different ${ }^{25} . »(S S, 26)$

Or, dans la mesure où cette dimension référentielle (extensive, reterritorialisante) de l'écriture de Scott implique l'irruption constante de l'altérité, elle est

$$
++
$$

18 "L'écoute permet, à la longue, de se défaire des limites identitaires rigides; le sujet peut se glisser dans le contexte.» 19 Sur ce point, voir Catherine Leclerc, "With the energy that follows une nuit blanche", ou du français dans la littérature anglo-québécoise», http://orees.concordia.ca/archives/numero2/essai/ catherineLeclerc.html, p. 4. 20 Traduction de: «find there are things they can't find the words for in English» (Linda Leith, op. cit., p. 24). 21 Traduction de: «a voice that is as close as I can get to my own experience, a voice which reflects in the very texture of the text, in the way the sentences and language sound, the point where the cultures cross.» (Gail Scott, «"a very rhythmic and almost conversational surface"», op. cit., p. 263) 22 Traduction de: "to raise the issue of how the English heroine (of a novel) might look against the backdrop of contemporary Québec [society]» (Gail Scott, Heroine, Vancouver, Talonbooks, 1999 [1987], p. 89). Désormais, les références à ce roman seront indiquées par le sigle $H$, suivi du folio, et placées entre parenthèses dans le texte. 23 Voir Beverley Daurio, «Interview with Gail Scott», Paragraph, vol. 15, n 2, 1993, p. 3. 24 «Je commence à comprendre l'influence de sa grammaire interne sur la phrase, le paragraphe, la manière dont les paragraphes, et les phrases, "glissent", pour refléter la turbulence de cette résonance intérieure.» 25 «On a tout simplement besoin de transcender cette peur d'avoir une voix... différente.» 
marquée à son tour par l'ouverture et l'intermédialité. Loin de renvoyer à des référents "pleins» et univoques, les divers points d'ancrage contextuel assurés par les effets de traduction déclenchent ce qu'elle appelle un bourdonnement d'associations interculturelles, une sorte de délire participatif chez le lecteur, qu'il sache ou non traduire les fragments rédigés en français. Partie intégrante du processus de minorisation, lequel implique un usage a-référentiel et a-signifiant (intensif) de la langue, ce bourdonnement composé de tonalités étrangères fait proliférer, entre les termes fondateurs des dichotomies traditionnelles (Québec/Canada, anglais/ français, soi/autrui et majeur/mineur), des lignes de fuite hors des références communes, selon une logique ayant moins à voir avec la métaphore (ressemblance) ou l'antithèse (dissemblance) qu'avec la métamorphose (devenir): «with the global predominance of English, I need a place to flee to ${ }^{26}$ » $(M Y, 8)$. Dans cette perspective, les effets de traduction et de bilinguisme dans les romans de Scott fonctionnent non seulement comme un trope de l'intermédialité, mais comme un lieu de production et de déploiement des lignes de fuite qui la constituent, indissociables d'une pratique narrative expérimentale qui s'efforce de créer des significations aux multiples points de croisement des langues et des cultures.

D'où la profonde ambivalence fonctionnelle des effets de traduction et de bilinguisme chez Scott, simultanément à la source de sa prose et engendrés par elle. Brouillant sans cesse les distinctions entre autoréférentialité (les effets de traduction s'avèrent des lieux d'inscription d'une réflexion sur l'acte d'écrire), référentialité (les effets de traduction sont des opérateurs de mimésis) et a-référentialité (les effets de traduction constituent des lieux intensifs où se déploient des sonorités et des rythmes a-signifiants), ils contribuent à ouvrir de nouveaux espaces intermédiaires qui dynamisent les frontières entre identités et langues pour affirmer la possibilité de leur devenir-autre. Car dans une conjoncture (post) moderne où domine le doute, les contradictions ne sont plus mutuellement exclusives, les reterritorialisations-déterritorialisations ne semblent jamais absolues, les égalités ne sont pas acquises une fois pour toutes. Bien au contraire, tout comme les rapports à l'altérité doivent être constamment (re)négociés et (ré)inventés, la différence doit être pensée selon des modalités multiples qui engagent aussi bien des processus d'identification et de "désidentification ${ }^{27}$ » que ceux de création. Du coup, affirmer les différences afin de lancer "un défi de plus au fade postulat d'analogie (c'està-dire on est pareil à (pense de la même façon que) l'autre dominant) qui est l'image de marque du patriarcat, ainsi que de toute culture dominante ${ }^{28} \gg(S S, 51)$ est aussi impératif que d'insister sur «ce que nous avons en commun [...], plutôt que ce qui nous sépare, rendant ainsi possible une forme d'écriture que nous n'avons pas

26 "étant donné la prédominance globale de l'anglais, j'ai besoin d'un lieu vers lequel je peux fuir ». 27 J'emprunte en le traduisant le terme «disidentification" proposé par Barbara Godard dans «Writing from the Border: Gail Scott on "The Main"», Lianne Moyes (dir.), Gail Scott. Essays on Her Works, Toronto, Guernica, 2002, p. 119. 28 Traduction de: "yet one more challenge to the bland assumption of sameness (i.e., one is (thinks) the same as the dominant other) which is the hallmark of patriarchy and dominant cultures everywhere». 
encore imaginée ${ }^{29}$.» $(M Y, 9)$ À partir du moment où l'on conçoit la différence comme «devenir-mineur", une telle posture de doute oblige à être à l'écoute non seulement de l'autre, mais de la porosité de la différence, dans le but de forger des stratégies de minorisation aptes à inscrire aussi bien le pluriel de l'altérité que sa mouvance, à savoir les multiples lignes de fuite hors des codes, lesquelles se croisent au sein de ce nouvel «espace interstitiel de différenciation doté d'un potentiel transformatif ${ }^{30}$ ».

\section{LES EFFETS DE TRADUCTION ET DE BILINGUISME DANS MY PARIS. A NOVEL}

Les effets de traduction et de bilinguisme dans Heroine et Main Brides relèvent en majeure partie d'une stratégie de minorisation et de non-traduction intratextuelle qui accorde au processus traductif le statut implicite - ambigu et risqué - de préalable nécessaire à l'écriture et à la lecture. En revanche, ceux de My Paris s'embrayent sur un travail intensif nettement plus radical et sur des modalités d'inscription du français extrêmement variées, où la traduction intratextuelle occupe une place prépondérante. Au lieu d'aménager, par l'abrupte apparition du français dans une phrase ou un paragraphe qui commence ou se termine en anglais, des pans d'altérité et d'opacité linguistiques qui dénotent, en plus d'une certaine illusion référentielle problématisée, des «rencontres tendues» («strained encounters $^{31}$ ») entre sujet traduisant-écrivant et sujets lisants (tant bilingues que monolingues), My Paris multiplie les points de rencontre et de résistance dans une tentative de «trouer» la langue anglaise, mais aussi la langue française, de même que cet espace mitoyen où s'articulent les différences culturelles. Si le projet esthétique et politique de Heroine et de Main Brides consiste à faire venir un lieu intermédiaire susceptible d'exprimer, entre autres, la double marginalité de Scott et de ses narratrices par rapport aux contextes canadien et québécois, celui de son dernier roman vise les diverses manières dont il est possible de "flâner ${ }^{32}$ " dans l'intermédialité en la travaillant de l'intérieur. Il s'agit de déconstuire la logique bilatérale - donc binaire - qui informe, d'une part, les concepts de bilinguisme officiel et de non-traduction et, d'autre part, certaines procédures de disjonction ou de dispersion, très présentes dans Heroine ${ }^{33}$, qui n'engagent que l'entre-deux.

$$
+++
$$

29 Traduction de: "what we have in common [...], instead of what separates us, permitting some kind of writing we haven't yet imagined». 30 Traduction de: «space of differentiation in the interstice with transformative potential» (Barbara Godard, op. cit., p. 128). 31 Ibid., p. 123. 32 Selon Scott, la narratrice de My Paris est une parodie du flâneur du dix-neuvième siècle. Voir Corey Frost, "Some Other Kind of Subject, Less Bounded", How2, vol. 1, n 4, 2000, n. p. http://www.scc.rutgers.edu/however/v1_4_2000/current/ workbook/index. html. 33 Ces procédures sont déjà moins omniprésentes dans Main Brides, où le multilinguisme et le multiculturalisme sont explicitement thématisés. Les tensions principales demeurent toutefois les rapports entre le Canada et le Québec, d'une part, et l'anglais et le français, de l'autre. Elles sont constitutives de la représentation du corps comme «androgyne, bilingue» («androgynous, bilingual»). Gail Scott, Main Brides, Toronto, Coach House Press, 1993, p.146. Désormais, les références à cet ouvrage seront indiquées par le sigle $M B$, suivi du folio, et placées entre parenthèses dans le texte. 
Dans cette optique, My Paris diffuse les éventuels «effets pervers» susceptibles de découler de juxtapositions typiques des répliques de la narratrice bilingue de Heroine ainsi que de celles de la plupart de ses amis francophones souvent unilingues ${ }^{34}$ :

$C^{\prime}$ est étrange, I had this dream. We were in a hotel in Halifax. You and I were pulling on a silver plate. One on each side. Il y avait une lutte terrible pour voir qui finirait par l'avoir. Finally I said: “Oké, take it. But you'll never know what to do with it. Tu vas gâcher la chance que ça représente ${ }^{35 . "}(H, 60)$

En introduisant des zones de résistance et de non-appropriation - «une langue a besoin de pouvoir pour survivre ${ }^{36}$ » $(M B, 152)$ - y compris pour le lecteur bilingue appelé à admettre les hésitations sémantiques et les glissements rythmiques provoqués par sa propre traduction de ces syntagmes, la stratégie de non-traduction offre au lecteur monolingue des espaces intensifs purs, parfaitement a-signifiants, destinés à n'agir que sur son affect («at some point, if you succeed with your [writing] project, you touch some kind of button or nerve that is so incredible, that nobody has touched before ${ }^{37}$ ») et à l'obliger à confronter ses rapports à l'autre.

Il reste que, d'un point de vue idéologique, une telle stratégie cautionne la mise en place implicite de deux catégories de lecteurs linguistiquement et culturellement «non poreuses», articulées sur l'opposition entre monolinguisme et bilinguisme. Si la non-traduction des fragments en français dans Heroine et Main Brides permet d'éviter les ponts et ainsi "d'écrire par-dessus l'abîme», depuis «les limites, le seuil, le mouvement entre individus, cultures, expressions et possibilités sexuelles ${ }^{38}$ ", Scott ne propose, à propos de ce qu'elle nomme le rapport d'adresse qui lie sujet-écrivant et sujet-lisant, aucun lieu mitoyen où les différences (le rapport de domination) entre actes de lecture bilingue et monolingue puissent se négocier. Ce faisant, la nontraduction favorise - à la manière de certaines législations issues du bilinguisme officiel - le maintien potentiel des inégalités qu'il importait de dénoncer. Scott va jusqu'à thématiser ce danger lorsque ses narratrices font des concessions ironiques au monolinguisme ou qu'elles parodient la traduction comme un acte unilatéral d'effacement/remplacement tantôt politiquement agressif, tantôt culturellement illusoire:

In the park downstairs was a demonstration of Québécois and Irish comrades. Their slogans read: DOWN WITH IMPERIALLY-IMPOSED CONSTITUTIONS. Except in French $^{39} .(H, 41)$

$$
+++
$$

34 Pour une analyse de l'inscription directe du français dans Heroine, voir Catherine Leclerc, loc. cit. 35 "C'est étrange, j'ai fait un rêve. Nous étions à un hôtel à Halifax. Toi et moi tirions sur un plateau en argent. Une de chaque côté. [...] Enfin j'ai dit: "Oké, prends-le. Mais tu ne sauras jamais qu'en faire." " 36 Traduction de: «language needs power to survive». 37 «à un moment donné, si votre projet [d'écriture] est réussi, vous touchez un bouton ou un nerf incroyable, que personne n'a encore touché» (Gail Scott, «In Conversation", Lianne Moyes, op. cit., p. 209). 38 Traduction de: "write over the top»; «the cusps, the threshold, the movement between individuals, cultures, expressions and possibilities of gender» (Corey Frost, loc. cit.). 39 "Au parc en bas il y a avait une manifestation de camarades québécois et irlandais. Leurs slogans disaient: DOWN WITH IMPERIALLY-IMPOSED CONSTITUTIONS. Sauf en français.» 
The sign-shop men, dressed in white, march past again. The problem being how to place their sign, which says ENSEIGNES SIMON, in conformity with the Frenchonly sign law, over the one which says SIMON'S SIGNS in English, Arabic, Hebrew. Given the new one is inadequate in $\operatorname{size}^{40}(M B, 51)$.

I noticed the QUÉBEC INDÉPENDANT graffiti had been painted over by STARVING DOGS EAT SHIT ${ }^{41} \cdot(H, 36)$

C'est précisément cet effet de non-porosité entre lecteurs monolingues et bilingues que les traductions «simultanées » du troisième roman de Scott désamorcent, avant de l'intégrer au vaste métacommentaire sur la négociation interlinguale et interculturelle qui parcourt l'ensemble du texte. Une première allusion à cette stratégie se trouve dans un article publié peu de temps avant la parution de My Paris, où Scott illustre sa nouvelle conception de la traduction-écriture d'une référence à un pont montréalais:

Walking east, now, on Ste. Catherine, the pont Jacques Cartier in front of us punctuates the horizon in the beginning of its long arc or comma over the river. I have come to think of the comma as representing the cusp of translation; the site of drifting identity. In my fake Paris diary My Paris (forthcoming), the comma separates the French words from their English translations ${ }^{42}$. $(M Y, 8)$

Cette allusion anticipe sur les deux passages de My Paris dans lesquels la «virgule de la traduction» assume le statut, autoréflexif, de trope:

Once I disappearing. Down rue du Bac. Not yet floating. Again. [...]. Autumn in air. Intuition always to be in Paris then. [...]. To write. To wander. Being subject with foreign queen on dollars. I.e. nostalgic. For distance. Of 19th-century novel. [...]. Simultaneously trying to grasp some point in future. By eclipsing I of certain early20th traveling republicans. Who having abolished comma. Drawing all of us. In portrait of about three words. But if comma of translation disappearing. What of French-speaking America remaining ${ }^{43}$.

$$
+++
$$

40 «Les hommes du magasin d'enseignes, vêtus de blanc, repassent. Le problème étant de réussir à placer leur enseigne, qui se lit ENSEIGNES SIMON, conformément à la loi exigeant l'emploi exclusif du français, par-dessus celle qui se lit SIMON'S SIGNS en anglais, arabe, hébreu. Compte tenu des dimensions inadéquates de la nouvelle». 41 "J'ai remarqué qu'on avait peinturé STARVING DOGS EAT SHIT par-dessus le graffiti QUÉBEC INDÉPENDANT.» $\mathbf{4 2}$ «En nous promenant vers l'est maintenant, sur la rue Sainte-Catherine, le pont Jacques Cartier qui est devant nous ponctue l'horizon au commencement de son long arc ou virgule pardessus le fleuve. J'ai fini par voir dans la virgule l'image du seuil de la traduction; le lieu de l'identité flottante. Dans mon faux journal intime My Paris (à paraître), la virgule sépare les mots en français de leurs traductions anglaises.» 43 «Une fois je disparaissant. Dans rue du Bac. Pas encore flottant. De nouveau. [...] Automne dans l'air. Intuition toujours faut être à Paris à cette époque. [...] Pour écrire. Pour flâner. Étant sujet avec reine étrangère sur dollars. C'est-à-dire nostalgique. Pour la distance. Du roman du 19e [...] Simultanément essayant d'appréhender un quelconque point dans l'avenir. En faisant s'éclipser Je de certains voyageurs républicains du 
Under glass canopy of métro she blurting. Homos worse than hétéros these days. [...] I saying nothing. Wanting to stay afloat. To stay out of categories. Moving back and forth. Across comma of difference. A gerund. Or gesture ${ }^{44} .(P, 107)$

Inséparable de l'emploi à la fois abondant et hautement équivoque du participe présent (gérondif), "forme modale impersonnelle qui "participe" de l'adjectif [...] et du verbe ${ }^{45}$ » et qui fait filer l'anglais en projetant le sujet dans cet espace désormais ouvert entre lui et l'objet, la virgule de la traduction s'avère saturée de valeurs esthétiques, idéologiques et éthiques. Comme le souligne Sherry Simon: «[t]he comma is [...] a space in-between, a space of blurred categories and undecidability. Here alternatives are suspended, multiple realities come together, differences coexist. This is the space of the act of translation ${ }^{46} \%$.

Rendue transparente grâce à une activité traduisante intratextuelle qui s'effectue à l'emplacement de la virgule, l'opacité introduite par l'insertion du français dans My Paris est immédiatement dissipée, de sorte que la virgule devient l'indice d'un travail d'accommodement interlingual et interculturel s'accomplissant sans cesse à même la surface narrative. Les effets de traduction et de bilinguisme acquièrent ici une matérialité qu'ils ne possédaient pas dans les deux premiers romans, où leur statut implicite leur conférait un caractère abstrait, voire idéalisé. Ils dénotent aussi un espace de cohabitation culturelle où lecteurs monolingues et bilingues, dorénavant sur un pied d'égalité, peuvent se (re)positionner les uns par rapport aux autres. Au lieu d'encourager, insidieusement, la passivité du lecteur monolingue, ou pire, son exclusion, My Paris l'incite à un «effort participatif, c'està-dire vraiment démocratique ${ }^{47} \gg(P, 32)$.

Les virgules de la traduction foisonnent dans $M y$ Paris et semblent, à première vue, se conformer à un seul schéma : mot ou expression en français + virgule + traduction anglaise: «Le ministère est par là, the ministry's over there» $(P, 13)$; « $[a] s$ lasse, dazed. From fatigue. As day of arrival ${ }^{48}$ » $(P, 16)$; «[p] ossibly sympathizer of Félquistes, Québec Liberation Front ${ }^{49}$.» $(P, 45)$ Or, on constate rapidement que ce schéma admet un certain bougé, d'abord sur le plan de la ponctuation où la virgule est loin d'être le marqueur unique de l'activité traduisante et de la différence culturelle. En effet, sont aptes à revêtir ce rôle aussi bien la barre oblique, les guillemets, le point

$$
+++
$$

début 20 . Qui ayant aboli la virgule. Faisant de nous tous. Portrait avec environ trois mots. Mais si virgule de la traduction disparaissant. Que restant de l'Amérique francophone.» (Gail Scott, My Paris. A Novel, Toronto, The Mercury Press, 1999, p. 49) L'utilisation du gras est faite par Scott. Désormais, les références à ce roman seront indiquées par le sigle $P$, suivi du folio, et placées entre parenthèses dans le texte. $\mathbf{4 4}$ «Sous coupole en verre du métro elle s'écriant. Homos actuellement pires que hétéros. [...] Je ne disant rien. Voulant rester à la surface. Rester à l'extérieur des catégories. Glissant d'un bord à l'autre. De la virgule de la différence. Un gérondif. Ou geste.» 45 Petit Robert. 46 «[1] a virgule est [...] un espace intermédiaire, un espace d'indécidabilité où les catégories se brouillent. Ici, les alternatives sont suspendues, des réalités multiples se rencontrent, les différences coexistent. C'est l'espace de l'acte de traduction» (Sherry Simon, "The Paris Arcades, the Ponte Vecchio and the Comma of Translation", Lianne Moyes, op. cit., p. 147-148). 47 Traduction de: "participatory, i.e. truly democratic effort». 48 «[a]ussi lasse, dazed. De fatigue. Que jour d'arrivée». 49 «[p]eut-être sympathisant des Félquistes, Québec Liberation Front.» 
et le point-virgule que la conjonction «or» (ou): «[o]ver pont au Change. Old moneychanging bridge ${ }^{50 » ~}(P, 91)$; « [c] onfusing rue Croix-des-Petits-Champs/Crossof-Little-Fields Street ${ }^{51}$ » $(P, 20)$; " outer store or shutter ${ }^{52}$ » $(P, 19)$. À de rares endroits, la ponctuation fait totalement défaut: «Past giant Chantier construction site $^{53} »(P, 101)$, tandis qu'à d'autres, le schéma de base s'alourdit de marqueurs supplémentaires, autant de variantes libres tels les italiques, les caractères gras ou les majuscules: «Christmas bûche or $\log ^{54}$ » $(P, 148)$; «Je haïs TOUTES LES FEMMES, I hate all women.» Le schéma admet également des permutations de l'anglais et du français: «outer blind or "store"» $(P, 12)$; «I'm not kidding. Sans blague.» $(P, 37)$

Plus significatif encore, ce bougé cautionne la disparition subite de l'équivalent anglais, ce qui entraîne de subtils brouillages référentiels, comme dans l'exemple suivant où il règne une confusion momentanée entre store/ «blind» et magasin/ «store»; confusion d'autant plus plausible que la narratrice observe souvent, à travers les lattes du store de son studio, la vitrine du magasin d'en face: «[...] looking through cracks in the store ${ }^{55} »(P, 84)$. Un corollaire interessant de cette dernière stratégie consiste à la faire coïncider avec une stratégie de minorisation de l'anglais: "Yesterday somnambulently crossing boulevard ${ }^{56}$ » $(P, 25)$; «[k]nowing when to regard them [les policiers]. As furniture ${ }^{57}$.» $(P, 13)$ Par ailleurs, au lieu de subitement disparaître, la traduction peut s'effectuer de manière (ludiquement) sélective: "Lyon parsleyed ham. Little ripening white cheeses called crottin, droppings. Crème fraîche. Crème brûlée. Crème anglaise ${ }^{58} »(P, 86)$, tout comme elle peut faire défaut d'entrée de jeu, rappelant provisoirement la stratégie dominante de Heroine et de Main Brides: "The concierge tearing from her little mahogany loge ${ }^{59}$ » $(P, 13)$; «C naturellement substituting crème fraîche ${ }^{60}$ » $(P, 73)$; «[d]esigned by architect from chez nous ${ }^{61} »(P, 71)$. Inversement, toujours à l'instar des deux premiers romans, de nombreux fragments en anglais - noms de boutiques, slogans, extraits de poèmes, dialogues - relèvent d'un travail de traduction préalable qui occulte l'original; procédé qui engendre à l'occasion un effet (parodique?) de défamiliarisation aux résonances surréalistes:

Thinking time to start wandering. [...] Towards Hermit's Well. Stone's throw from Buffoon Street. Down Beaujolais Alley. Through Wolf's Crack or Breach. Hot Cat Road. Passage of Desire. Magenta Boulevard. Where walking Nadja toward Hôtel Sphinx ${ }^{62} .(P, 25-26$.

\footnotetext{
50 «[t]raversant pont au Change. Vieux money-changing bridge». 51 «[c]onfondant rue Croix-des-PetitsChamps/Cross-of-Little-Fields Street». $\mathbf{5 2}$ «store ou shutter extérieur». $\mathbf{5 3}$ «Passant devant Chantier géant construction site». 54 «Bûche ou log de Noël». 55 «[...] regardant à travers lattes du store». $\mathbf{5 6}$ «Hier traversant somnambulement le boulevard.» Je souligne. $\quad \mathbf{5 7}$ «Sachant quand les [policiers] prendre. Pour des meubles.» Le verbe anglais «to regard» signifie simultanément «to consider» (usage correct) et «to look at» (usage mineur sous l'influence de «regarder»). $\quad \mathbf{5 8}$ «Jambon de Lyon. Petits fromages blancs en train de mûrir appelés crottin, droppings.» 59 «La concierge se précipitant de sa petite loge en acajou». $\mathbf{6 0}$ "C naturellement y substituant de la crème fraîche». 61 «[c]onçu par architecte de chez nous». 62 «Pensant c'est moment de commencer à flâner. [...] Vers Puits de l'Hermite. Tout proche de rue Buffon. Passant par Impasse Beaujolais. A travers Crack ou Brèche du Loup. Rue du Chat. Passage du désir. Boulevard Magenta. Où Nadja marchant vers Hôtel Sphinx.»
} 
À partir de là, les variations se mettent à proliférer, déclenchant des perturbations, dérapages ou transformations qui, additionnés aux précédents, représentent non seulement la diversité des modalités selon lesquelles il est possible de dénoter l'altérité linguistique, mais aussi la diversité des politiques traductives à la disposition de tout sujet traduisant-écrivant. Complémentaires, contradictoires ou antagonistes, ces politiques consolident la visée proprement éthique des effets de traduction et de bilinguisme dans My Paris en faisant éclater les binarismes et en démultipliant les lignes de fuite au sein de l'espace interlingual créé par la virgule de la traduction et ses multiples substituts.

Plus spécifiquement, lorsqu'un fragment en français est suivi de sa traduction anglaise, celle-ci est chaque fois tributaire d'une éthique traductive précise, allant du littéralisme le plus strict (par exemple, la traduction des noms de rues) à l'adaptation la plus radicale ( At du Bac/Saint-Germain/Raspail. The dock. The saint. The general ${ }^{63}$ » $\left.[P, 98]\right)$, en passant par divers processus plus ou moins annexionnistes, dont la traduction-résumé («Fort éprouvé par la maladie, sick» $[P, 68]$ ), la paronomase («ennervée, unnerved» $[P, 157]$; «femmes-cuir (queer), leatherwomen» $[P, 121])$, l'erreur volontaire («loge, cage» $[P, 117]$ ) et l'effacement de l'original. C'est ainsi que la stratégie de traduction se double souvent d'une explication-explicitation, laquelle peut revêtir plusieurs formes et présupposer diverses postures éthiques qui, à la limite, coexistent à l'intérieur d'un même fragment ${ }^{64}$ :

Tapant la balle comme d'habitude, marking time (bouncing ball) as usual. $(P, 70)$

Seeping problèmes de voisinage. Article saying euphemistically. I.e. lack of privacy $^{65} \cdot(P, 93)$

Being décalée, time-gapped. As French calling jet-lagged ${ }^{66} .(P, 16)$

To left - law faculty. LIBERTÉ. ÉGALITÉ. FRATERNITÉ - dictum of French Revolution ${ }^{67}$. $(P, 110)$

Sitting in café at Sèvres-Babylone. I.e. at corner of Exquisite China and Pursuit of Sensuous Pleasure ${ }^{68} .(P, 25)$

And I wrapped in blankets with my grippe carabinée, rifling cold. To use a term from chez nous ${ }^{69} .(P, 25)$

$$
+++
$$

63 «[...] Le Quai. Le Saint. Le Général». 64 C'est le cas des extraits un, trois, cinq et six qui suivent, où le littéralisme (ex. carabiné = rifling) se combine, de manière contradictoire, avec l'explication. Voir Antoine Berman, L'épreuve de l'étranger, Paris, Éditions Gallimard, 1984. 65 "Problèmes endémiques de voisinage. Article disant euphémiquement. C'est-à-dire absence d'intimité.» 66 "Étant décalée, time-gapped. Comme disent Français pour jet-lagged.» $\mathbf{6 7}$ "À gauche - faculté de droit. Liberté. Égalité. Fraternité - dicton de la Révolution française.» 68 "Assise dans café à Sèvres-Babylone. C'est-à-dire au coin de Porcelaine Exquise et Poursuite de Plaisirs Sensuels.» 69 «Et je enveloppée de couvertures avec ma grippe carabinée, rifling cold. Pour employer un terme de chez nous.» 
Ces extraits sont particulièrement intéressants dans la mesure où, pris ensemble, ils préfigurent, selon un mode ironique, des lecteurs différents, dont un lecteur non français (à qui il faut expliquer l'origine du slogan liberté-égalité-fraternité), un lecteur québécois dont le français (grippe = cold) n'est pas exactement le même que celui des Français, un lecteur français et un lecteur canadien-anglais bilingue à qui il faut signaler (ou rappeler) la spécificité du franco-québécois, ainsi qu'un lecteur anglophone monolingue pour qui il faut tout traduire et un lecteur anglophone (ou francophone) bilingue n'ayant pas besoin de traduction. Or, loin de correspondre à des catégories étanches et hiérarchisées, ces lecteurs sont poreux, glissants, parodiques, dépourvus de la consistance (unicité, uniformité, présence à soi) nécessaire pour s'imposer comme sujets pleins et autonomes. La rapidité avec laquelle ils alternent dans les interstices des langues et des cultures leur confère une hybridité mobile qui les rend irréductibles aux dualismes et aux dichotomies, si bien que la frontière entre monolinguisme et bilinguisme se révèle être aussi éphémère que non localisable.

Nous avons affaire ici à ce que Scott a nommé "l'insaisissabilité surdéterminée du $[\mathrm{moi}]^{70}$ » $(S S, 88)$ qui caractérise non seulement le moi (en l'occurrence, les lecteurs implicites), mais aussi toute entité culturelle et linguistique qui se donne pour monolithique, y compris la langue française. En effet, en plus de poursuivre, en le radicalisant, le projet de minorisation de l'anglais inauguré par Heroine, $M y$ Paris contient de nombreux indicateurs de québécité - le plus fréquent étant l'emploi de l'expression (jamais traduite) "chez nous», aux connotations "étrangères» évidentes en contexte parisien - qui ont pour effet de minoriser à tour de rôle le français standard et le franco-québécois. Certains de ces indicateurs, comme ceux ayant trait à l'accent, possèdent un statut thématique et servent à commenter la différence entre intonations parisiennes, québécoises et anglo-québécoises. D'autres, comme nous venons de le voir, possèdent un statut hybride, à mi-chemin entre le thème et l'effet de traduction: «Laughing uproariously when I asking where to put vidange. As we saying chez nous. For garbage. Here vidange meaning sloppail. Should have said poubelle ${ }^{71}$ » $(P, 41)$; «[a]t the same time watching the québécoise C's lovely full lips. [...] Mocking French mocking québécois way of speaking. Nasalizing pain, $\operatorname{bread}^{72} »(P, 31)$; «[t]he rosy Breton waitress bursting out. As I ordering vi-h-n, wine: O la petite cousine québécoise, the little Québec cousin ${ }^{73} »(P, 62)$.

En dernière analyse, les effets de traduction dans My Paris illustrent le caractère ambigu, insaisissable et indécidable non seulement des identités et des langues, mais de l'altérité elle-même, dans la mesure où la cohabitation implique moins un phénomène statique de juxtaposition qu'un processus kaléidoscopique de

70 Traduction de: «the overdetermined illusiveness of [self]». 71 «Riant à gorge déployée quand je demandant où mettre vidange. Comme nous disant chez nous. Pour déchets. Ici vidange signifiant seau d'eau sale. Aurais dû dire poubelle». $\mathbf{7 2}$ «[e]n même temps regardant les belles lèvres pulpeuses de la Québécoise C. [...] Se moquant des Français se moquant du parler québécois. Nasalisant pain, bread». 73 «[l]a serveuse bretonne aux joues roses s'écriant. Quand je commandant vi-h-n, wine». 
chevauchements, de déterritorialisations-reterritorialisations sans cesse amorcés, jamais tout à fait achevés: "[...] I feeling in middle. Neither One. Nor entirely bleeding into context either ${ }^{74} \gg(P, 31)$. L'altérité n'est ni un état qu'il s'agit de (s')assigner ni une entité ou un site qu'il s'agit de nommer; elle relève plutôt d'une dynamique interstitielle qui empêche les codes d'advenir grâce aux glissements provoqués par les lignes de fuite qu'elle libère sous forme d'options de traduction disparates. Ouvrant la différence de l'intérieur, ces lignes de fuite font éclater l'espace trop étroit de l'entre-deux, fondé sur une logique de l'assimilation et de la dissimilation, afin de rendre possibles des négociations identitaires, culturelles et linguistiques multilatérales entre les diverses facettes de soi et de l'autre:

\section{[...] I am of the American continent, but not American. Canadian? Yes and no. Québécois(e)? A bizarre oft-repeated event during my stay in Paris: I open my mouth to speak and I am a Québécois "cousin"; then an English inflection creeps into my speech, and I'm Other ${ }^{75}$. $(M Y, 8)$}

C'est dire que My Paris déplace et complexifie les enjeux soulevés par Heroine et Main Brides, où les passages en français fonctionnent à la manière d'un palimpseste, trace ambiguë d'un processus interlingual et interculturel tantôt achevé, tantôt à venir. En recourant à la virgule de la traduction (de la différence), Scott attribue aux effets de traduction et de bilinguisme une matérialité et une fonctionnalité qui lui permettent de déjouer les effets pervers susceptibles de découler de la stratégie de non-traduction, dès lors perçue, à l'instar de la virgule de la traduction elle-même, comme une posture éthique parmi d'autres, c'est-à-dire comme une manière, en soi équivoque et risquée, de négocier les rapports à l'altérité. Car s'il est urgent d'afficher les différences pour ne pas disparaître, il faut récuser, par le biais d'un projet esthétique et politique de minorisation, la conception libérale de la cohabitation pacifique des langues et des cultures qui occulte les effets de pouvoir et de domination. Et s'il est tout aussi urgent de reconnaître les similitudes culturelles et identitaires pour être en mesure de déconstruire les polarités qui caractérisent une volonté de différenciation trop intransigeante, il faut se prémunir contre "le danger d' "uniformités" fausses ou simulées. Issues de certaines notions républicaines d'égalité ${ }^{76}$.» $(P, 90) D^{\prime}$ où la nécessité de donner droit de cité à une gamme d'options traductives qui inclut, sans toutefois s'y limiter, l'assimilation (identification), la cohabitation, la résistance, l'affrontement, la dissimilation (désidentification) et le jeu, en les insérant dans une suite d'égalités («string of equalities») qui libère sans niveler, brouille sans effacer, mélange sans susciter de nouveaux effets de hiérarchie.

74 «je me sentant au milieu. Ni Une. Ni m'immisçant complètement dans contexte non plus». $\mathbf{7 5}$ «je suis du continent américain, mais ne suis pas américaine. Canadienne? Oui et non. Québécoise? Un événement bizarre qui s'est souvent répété pendant mon séjour à Paris: j'ouvre la bouche pour parler et je suis une “cousine" québécoise; puis une inflexion anglaise se glisse dans mes paroles, et je suis Autre.» 76 Traduction de: «dangerous fake or feigned "uniformities." Arising from certain republican notions of equality.» 
Il s'ensuit que la posture de doute se double ici d'une posture éthique qui définit la différence culturelle non pas comme une essence à conserver ou à rejeter, mais comme un lieu multiforme et poreux dont on peut constamment redessiner les contours. À cet égard, My Paris peut se lire comme un métacommentaire implicite sur la capacité de la traduction à forger, à partir des carrefours mouvants aménagés par la virgule de la traduction et ses variantes, de nouvelles images de soi et de l'autre, pour peu que le sujet traduisant-écrivant - accompagné de ses lecteurs - accepte de «flâner» dans les interstices glissants des langues et des cultures. 